\section{MS27-P5 Accurate structure refinement of thin films using 3D electron diffraction data}

Gwladys Steciuk ${ }^{1}$, Lukas Palatinus ${ }^{2}$, Philippe Boullay ${ }^{1}$, Adrian David $^{1}$, Morgane Lacotte ${ }^{1}$, Olivier Copie ${ }^{1}$, Wilfrid Prellier ${ }^{1}$, Helène Rotella ${ }^{1}$

1. CRISMAT, CNRS UMR 6508, 6 Bd du Maréchal JUIN 14050 CAEN Cedex, France

2. Institute of Physics of the AS CR, v.v.i. Na Slovance 2, 18221 Prague, Czechia

email: gwladys.steciuk@ensicaen.fr

2D nanostructures emerge as one leading topic in fundamental materials science where transition metal oxides appear as promising candidates because of their strong interplay between charge, spin, orbital and lattice. Low distortions or size effects due to substrate strain and reduced dimension of the film could induce drastic changes in the observed properties between a thin film material and its bulk analogous. In most cases, the challenge for thin film materials is not to solve the structure, which is generally known, but the structure analysis should be accurate enough to account for these small variations.

Regarding the data collection, Precession Electron Diffraction Tomography (PEDT) aims to collect a series of randomly oriented diffraction patterns at a fixed angular interval. It is similar to the phi-scan data collection used in single crystal X-ray diffraction (XRD) but applicable to objects of a few tens of nanometers. Not limited by the geometry of the sample, the PEDT data collection allowed to access a much larger number of reflections than one would ever obtain using reciprocal space maps acquired with a high-resolution diffractometer [1]. Still, unlike the case of XRD, the kinematical theory of diffraction is not valid for electrons. By limiting the paths for multiple scattering, PEDT allows to reduce dynamical effects but not enough to consider PEDT data as kinematical. To tackle this problem, the recent implementation of refinements based on the dynamical diffraction theory in JANA2006 [2] is very promising.

In this contribution, we will present our first results on the use of the dynamical diffraction theory for the accurate structure refinement of perovskite related compounds from PEDT data. First, the result of the structure refinement considering the kinematical approximation and the dynamical theory will be presented for PrVO, a compound with a distorted perovskite structure. The results obtained for bulk-PrVO will be compared against the solution from neutron diffraction illustrating the gain of reliability achieved using the dynamical theory. Second, we will use and discuss the interest and limitation of this new crystallographic tool to refine the structure of perovskite related thin films.

[1] H. Rotella, et al, J. Phys.: Condens. Matter 27 (2015) 175001.

[2] L. Palatinus et al, Acta Cryst. A71 (2015) 235.

Keywords: precession electron diffraction tomography, structure refinement, thin film

\section{MS27-P6 Electron crystallography of 3D nano-crystals}

Max T.B. Clabbers ${ }^{1}$, Eric van Genderen ${ }^{1}$, Igor Nederlof ${ }^{1,2}$, Yao-Wang $\mathrm{Li}^{1}$, Jan Pieter Abrahams ${ }^{1}$

1. Leiden University, Biophysical Structural Chemistry, Einsteinweg 55, 2333CC Leiden, The Netherlands

2. Amsterdam Scientific Instruments, Science Park 105, 1098XG Amsterdam, The Netherlands

email: clabbersm@gmail.com

Many proteins do not form crystals large enough and of sufficient quality for 3D structure determination by X-ray crystallography. For such sub-micron sized 3D crystals there are several alternatives: intense XFEL sources, electron imaging and electron diffraction. Solving new structures using these approaches remains a problem as ab-initio crystal phasing is hampered by experimental constraints that severely compromise data quality. These constraints include incomplete sampling of Bragg spots and (for electron diffraction), dynamic and inelastic scattering.

In case of electron diffraction, complete sampling of Bragg spacings can be achieved with a fast Timepix quantum area detector. This detector is of unprecedented sensitivity and combined with continuous crystal rotation electron diffraction data can be collected under low dose conditions. From a single frozen lysozyme nano-crystal of only $200 \mathrm{~nm}$ thin a rotation series of 40 degrees was collected with very fine slicing showing diffraction up to $2 \AA$ resolution. Furthermore, in case of small pharmaceutical compounds, a complete dataset could be collected at room temperature from nano-crystals of only $200 \mathrm{~nm}$ thin showing Bragg spots up to $0.8 \AA$ resolution. Phasing and structure solution for these pharmaceuticals was possible using direct methods yielding a structural model at atomic resolution.

Ab-initio phase information can be measured directly by electron imaging of protein nano-crystals. This experimental approach allows enhancing the resolution of electron images of $3 \mathrm{D}$ nano-crystals to beyond $2 \AA$ including crystal phases, by separating mosaic blocks within nano-crystals through image processing.

Keywords: Electron crystallography, nano-crystals, quantum area detector 